\title{
Cesarean delivery and small newborn for gestational age
}

\author{
Cesariana e recém-nascido Pequeno PARA a idAde gestacional
}

Authorship: Federação Brasileira das Associações de Ginecologia e Obstetrícia (Febrasgo)

Participants: Ricardo Simões ${ }^{1,2}$, Wanderley M. Bernardo ${ }^{2}$, Antônio J. Salomão ${ }^{1}$, Edmund C. Baracat ${ }^{1}$

Conflict of interest: none

${ }^{1}$ Federação Brasileira das Associações de Ginecologia e Obstetrícia (Febrasgo)

${ }^{2}$ Guidelines Project, Associação Médica Brasileira

The Guidelines Project, an initiative of the Brazilian Medical Association, aims to combine information from the medical field in order to standardize procedures to assist the reasoning and decision-making of doctors.

The information provided through this project must be assessed and criticized by the physician responsible for the conduct that will be adopted, depending on the conditions and the clinical status of each patient.

\section{INTRODUCTION}

Small for gestational age (SGA) refers to a fetus that has failed to reach a specific biometry, including abdominal circumference consistent with gestational age, which may be caused by prematurity, intrauterine growth restriction, or both, with variable etiology and effects.

The definition of small for gestational age (SGA) newborn is not clear. According to the World Health Organization (WHO), SGA is defined as a fetus whose weight at birth is below the 10 percentile for its gestational age and gender, based on an intrauterine growth curve ${ }^{1}(\mathbf{D})$. However, this definition has the main disadvantage of depending on the weight curve at birth that is used as a reference, although it varies according to the population studied. Another definition considered for SGA newborns is the one endorsed by the International Society of Pediatric Endocrinology, which considers small for gestational age infants born with weight and/or length less than two or more standard deviations of the mean for gestational age. Other authors also consider SGA infants born at term and whose birth weight is $\leq 2,500 \mathrm{~g}$.

Exception should be made with respect to the concept of SGA, used as an indicator of intrauterine growth restriction, terms that should never be applied interchangeably since some babies considered small for gestational age may simply represent those located in the lower portion of a normal distribution curve for fetal growth, as in the case of genetic predisposition (parents are small).

It is known that the birth weight is the most important factor in neonatal mortality, and also a significant determinant of post-natal mortality and infant morbidity. This may explain the high morbidity and mortality rates identified among infants born small for gestational age, compared to those observed for newborns consid- ered appropriate for gestational age $(\mathrm{AGA})^{2}(\mathbf{B})$. Thus, better management of the health of these children is imperative, starting with prenatal care and delivery assistance.

\section{Objective}

The objective of this review is to provide the best evidence available today for cesarean section indication in pregnancies with small for gestational age fetuses, and its effect on perinatal and neonatal morbidity and mortality.

\section{Material and methods}

The evidence to be used for analysis of perinatal and neonatal morbidity and mortality, according to the mode of delivery chosen for pregnancies with SGA fetuses, was obtained based on the following steps: preparation of the clinical question, structuring of the question, search for evidence, critical evaluation, and evidence selection.

\section{Clinical question}

Is cesarean delivery in pregnancies with small for gestational age fetuses related to lower perinatal and neonatal morbidity and mortality compared to vaginal delivery?

\section{Structured question}

The clinical question is structured according to the P.I.C.O. components (P (Patient); I (Intervention); C (Comparison); O (Outcome)).

- P: Small for gestational age.

- I: Cesarean-section.

- C: Vaginal deliver.

- O: Perinatal e neonatal morbidity and mortality. 
Bases of scientific data consulted

The scientific databases consulted were: PubMed-Medline and Cochrane. A manual search from the references of reviews (narrative or systematic) was also performed.

Strategies for search of evidence

PubMed-Medline

Strategy: (Cesarean Section OR Cesarean Sections OR Delivery, Abdominal OR Abdominal Deliveries OR Deliveries, Abdominal OR Caesarean Section OR Caesarean Sections OR Abdominal Delivery OR C-Section OR C Section OR C-Sections OR Postcesarean Section) AND (Infant, Small for Gestational Age).

\section{Cochrane}

Strategy: cesarean section AND Small for Gestational Age.

Studies retrieved (7/17/14) (Table 1)

TABLE 1 Number of studies retrieved with the search strategies used for each scientific database.

\begin{tabular}{ll} 
Database & Number of studies \\
\hline Primary & \\
\hline PubMed-Medline & 623 \\
\hline Cochrane & 114
\end{tabular}

Inclusion criteria for studies retrieved

Selection of studies, assessment of titles and abstracts obtained from the search strategy in the consulted databases was conducted by two researchers with skills in the preparation of systematic reviews, both independent and blinded, strictly observing the inclusion and exclusion criteria previously established (see item "P.I.C.O components"). All potentially relevant studies were identified. Whenever the title and the summary were not enlightening, researchers sought the full article.

\section{Study desiǵn}

Narrative reviews, case reports, case series and studies presenting preliminary results were excluded from the assessment. Systematic reviews and meta-analyzes were used with the basic purpose of recovering references that perhaps had been lost at first, from the initial search strategy. Studies designed as cohort or controlled clinical trials (randomized or not) were included.

Cohort studies were defined as those with follow-up of patients, the same history, and analysis of prognostic outcomes.
Controlled clinical trials were evaluated according to the Jadad score. ${ }^{3}$

P.I.C.0. components

- Patient: pregnant women (nulliparous and multiparous) with singleton small for gestational age fetuses in cephalic position.

- Intervention: cesarean-section.

- Comparison: vaginal delivery.

- Outcome: newborn outcomes included: perinatal or neonatal death (excluding cases of death related to fatal fetal abnormalities), neonatal morbidity, such as seizures (occurring within the first 24 hours of birth or that require two or more drugs to control), Apgar score, birth asphyxia, respiratory complications, infection, need for admission into neonatal intensive care unit, neonatal encephalopathy, trauma at birth (bone fractures, subdural hematoma, cerebral or intraventricular hemorrhage), spinal cord injury, peripheral nerve injury (e.g., brachial plexus injury), disabilities in childhood, hypotonia, intubation or need for ventilation for at least 24 hours, and need for tube feeding for four days or longer.

\section{Language}

We included studies available in Portuguese, English, French or Spanish.

\section{According to publication}

Only studies with full text available were considered for critical assessment.

\section{Studies selected in the first assessment}

After entering the search strategy in the primary databases (PubMed-Medline and Cochrane), the assessment of titles and abstracts led to the selection of three studies.

Evidence selected in critical evaluation and exhibition of results The studies considered for full text reading were critically assessed according to inclusion and exclusion criteria, study design, P.I.C.O., language and availability of the full text.

Results pertaining clinical status will be displayed individually showing the following items: clinical question, number of studies selected (according to inclusion criteria), description of the studies (Table 2), results and summary of the available evidence.

References related to the studies included are shown in Table 4, and are also presented in the section References. 
After applying the inclusion and exclusion criteria, the evidence selected in the search and defined as randomized controlled trials (RCT) were subjected to an appropriate checklist for critical assessment (Table 3). Critical assessment of RCTs allows to classify them according to the Jadad score, so that Jadad < three (3) trials are considered inconsistent (grade $\mathbf{B}$ ), and those with scores $\geq$ three (3), consistent (grade A). For critical analysis of nonrandomized studies, among them prospective observational studies, we used the Newcastle-Ottawa scale. ${ }^{4}$

For results with available evidence, wherever possible the following specific items are defined: population, intervention, outcomes, the presence or absence of benefit and/or damage and controversies.

The results will be presented preferably in absolute data, absolute risk, number needed to treat (NNT), or number needed to harm (NNH), and occasionally in mean and standard deviation.

\section{TABLE 2 Worksheet used for description of studies} included and exposure of the results

Worksheet for description of studies and exposure of the results

\begin{tabular}{|c|c|}
\hline \multicolumn{2}{|l|}{ Evidence included } \\
\hline \multicolumn{2}{|l|}{ Study design } \\
\hline \multicolumn{2}{|l|}{ Population selected } \\
\hline \multicolumn{2}{|l|}{ Time of follow-up } \\
\hline \multicolumn{2}{|l|}{ Outcomes considered } \\
\hline \multicolumn{2}{|c|}{ Expression of results: percentage, risk, odds, hazard ratio } \\
\hline \multicolumn{2}{|c|}{$\begin{array}{l}\text { TABLE } 3 \text { Critical assessment script for randomized } \\
\text { controlled trials (checklist). }\end{array}$} \\
\hline Study data & Sample size calculation \\
\hline $\begin{array}{l}\text { Reference, study design, Jadad, } \\
\text { strength of evidence }\end{array}$ & $\begin{array}{l}\text { Estimated differences, power, signifi- } \\
\text { cance level, total number of patients }\end{array}$ \\
\hline Patient selection & Patients \\
\hline Inclusion and exclusion criteria & $\begin{array}{l}\text { Recruited, randomized, prognostic } \\
\text { differences }\end{array}$ \\
\hline Randomization & Patient follow-up \\
\hline $\begin{array}{l}\text { Description and blinded } \\
\text { allocation }\end{array}$ & Time, losses, migration \\
\hline Treatment protocol & Analysis \\
\hline $\begin{array}{l}\text { Intervention, control and } \\
\text { blinding }\end{array}$ & $\begin{array}{l}\text { Intention to treat, analyzes of } \\
\text { intervention and control }\end{array}$ \\
\hline Outcomes considered & Result \\
\hline $\begin{array}{l}\text { Primary, secondary, measuring } \\
\text { instrument of the outcome of } \\
\text { interest }\end{array}$ & $\begin{array}{l}\text { Benefit or harm in absolute data, } \\
\text { benefit or harm on average }\end{array}$ \\
\hline
\end{tabular}

\section{RESULTS}

Clinical question

Is cesarean delivery in pregnancies with small for gestational age fetuses related to lower perinatal and neonatal morbidity and mortality compared to vaginal delivery?

\section{TABLE 4 Selection process.}

\section{Evidence selected}

Type of publication

Non-concurrent cohort studies

Included

$3^{5-7}$

The main reasons for the exclusion of works were: the unavailability of the full text; a study design other than longitudinal observational (retrospective or prospective) or experimental (controlled clinical trials, randomized or not) studies.

Results of the evidence selected

Of the 737 articles initially retrieved, 3 were selected to support the summary of evidence concerning perinatal and neonatal morbidity and mortality, according to mode of delivery chosen for pregnancies of small for gestational age fetuses. Studies included are shown in Table 4 .

1. Kinzler WL, et al. (B) ${ }^{5}$

- Design: non-concurrent observational longitudinal study (1995 to 1997).

- Population: women who gave birth to small for gestational age babies, in cephalic presentation and with gestational age between 24-42 weeks.

- Outcome: to evaluate neonatal morbidity and mortality among infants exposed and not exposed to labor.

- Result: of the 986,405 small for gestational age newborns, $87.4 \%$ were exposed to labor. Of these, fetuses with gestational age from 24 to 31 weeks had the highest risk of death in the early neonatal period compared to those born by cesarean section. However, analyzing the late neonatal and postnatal period, the authors found that for fetuses subject to labor there was a lower risk of death at all gestational ages.

2. Boers KE, et al. (B). ${ }^{6}$

- Design: non-concurrent observational longitudinal study. 
- Population: retrospective analysis of all births of fetuses with gestational age $>36$ weeks occurred between the years 2000-2004.

- Outcome: neonatal morbidity and mortality.

- Result: a total of 14,416 births of babies weighing less than the $10^{\text {th }}$ percentile for gestational age were identified. In this study, the authors found no difference in neonatal outcomes (neonatal mortality, and Apgar score <7) between fetuses born by spontaneous vaginal delivery or elective cesarean delivery ( $R R=0.44$ with 95CI: 0.02 to 3.0 and $R R=0.97$ with 95 CI: 0.61 to 1.5 , respectively).

3. Lee HC, et al. (B) ${ }^{\text {? }}$

- Design: non-concurrent observational longitudinal study.

- Population: retrospective analysis of all births that occurred between the years 1999-2000. Newborns with gestational age from 26 to 36 weeks were considered small for gestational age when their weight was below the $10^{\text {th }}$ percentile (SGA newborns), or appropriate for gestational age (AGA) when their weight was between the $10^{\text {th }}$ and $90^{\text {th }}$ percentiles.

- Outcome: neonatal morbidity and mortality according to the mode of delivery selected.

- Result: in this study, there was a higher cesarean section rate among SGA newborns compared to those considered AGA. These rates were more prominent among fetuses with gestational ages from 26 to 32 weeks (50 to 67\%) while those considered AGA had cesarean rates ranging from 22 to $38 \%$. Newborns considered small for gestational age at week 31 of pregnancy had increased survival with cesarean section, while those found small for gestational age at week 33 or later, as well as AGA infants had decreased survival when delivery was performed through cesarean section.

\section{Discussion}

In this review, the authors found no prospective observational studies examining the neonatal outcomes in question, regarding the selected mode of delivery (cesarean or vaginal) for pregnancies of small for gestational age fetuses. However, retrospective studies analyzing these outcomes are described. The methodological limitations intrinsic to these designs should be taken into consideration, especially with regard to the heterogeneity of the population included in the studies, which is a very important factor since the very definition of SGA varies according to the population curve adopted. Moreover, we noticed in the studies retrieved the association of another risk factor related to neonatal morbidity and mortality, which is prematurity. Although only SGA fetuses were included, it is possible that this could have introduced bias into the analysis of morbidity and mortality related to the mode of delivery, since many small for gestational age newborns are not premature and therefore they have no problems connected to prematurity.

A retrospective study assessed in this analysis suggests that small for gestational age newborns (between 24-31 weeks) exposed to labor have increased risk of early neonatal death compared with those not subject to labor. On the other hand, in this same analysis, the authors identified a lower risk of death in the late neonatal and postnatal periods to those born vaginally, regardless of gestational age ${ }^{5}(\mathbf{B})$. Contradictory results were found in another retrospective study, which showed that SGA neonates born at 31 weeks had lower late neonatal mortality with cesarean delivery, while SGA newborns with gestational age > 33 weeks, as well as those considered AGA, had higher mortality rates associated with cesarean sec$\operatorname{tion}^{7}(\mathbf{B})$.

Another study concluded that there was no difference in neonatal mortality and morbidity according to Apgar score between fetuses born vaginally and those delivered by elective cesarean section ${ }^{6}(\mathbf{B})$.

\section{Final ReCOMmendations}

In the absence of other obstetric indications requiring cesarean section, there is no sufficient evidence to recommend planned cesarean section in the pregnancy of small for gestational age fetuses aiming to reduce neonatal and perinatal morbidity and mortality.

\section{References}

1. Intrauterine growth retardation in newborn children. Available from: http:// www.who.int/ceh/indicators/iugrnewborn.pdf. Acessado em: 10.jul.2014.

2. Clausson B, Cnattingius S, Axelsson O. Preterm and term births of small for gestational age infants: a population-based study of risk factors among nulliparous women. Br J Obstet Gynaecol. 1998; 105(9):1011-7. PubMed PMID: 9763054.

3. Jadad AR, Moore RA, Carroll D, Jenkinson C, Reynolds DJ, Gavaghan DJ, et al. Assessing the quality of reports of randomized clinical trials: is blinding necessary? Control Clin Trials. 1996; 17:1-12.

4. Wells GA, Shea B, O'Connell D, Peterson J, Welch V, Losos M, et al. The Newcastle-Ottawa Scale (NOS) for assessing the quality of nonrandomised studies in meta-analyses. Available from: www.ohri.ca/programs/clinical_ epidemiology/oxford.asp. Acessado em: 10.fev.2014. 
5. Kinzler WL, Ananth CV, Smulian JC, Vintzileos AM. The effects of labor on infant mortality among small-for-gestational-age infants in the USA. Matern Fetal Neonatal Med. 2002; 12(3):201-6. PubMed PMID: 12530619.

6. Boers KE, van der Post JA, Mol BW, van Lith JM, Scherjon SA. Labour and neonatal outcome in small for gestational age babies delivered beyond $36+0$ weeks: a retrospective cohort study J Pregnancy 2011; 2011:293516. PubMed PMID: 21490789.

7. Lee HC, Gould JB. Survival rates and mode of delivery for vertex preterm neonates according to small- or appropriate-for-gestational-age status. Pediatrics. 2006; 118(6):e1836-44. PubMed PMID: 17142505. 